\title{
多尺度卷积与动态权重代价函数的全卷积网络工业烟尘目标分割
}

\author{
刘辉, 张俊鹏 ${ }^{*}$, 李清荣 \\ (昆明理工大学信息工程与自动化学院 昆明 650500) \\ (1318843416@qq.com)
}

\begin{abstract}
摘 要: 烟尘黑度是工业污染监测中的一项重要指标, 针对用计算机图像识别技术在基于林格曼烟气黑度烟尘自动 监测中如何将烟尘目标区域从背景中有效分割, 以及工业烟尘具有形状不固定和云相似度高等特点, 导致现有方法 在复杂场景下分割烟尘的结果不准确，提出了一种改进全卷积网络模型. 结合多尺度卷积操作，增强网络的特征提 取能力; 通过在交叉熵的基础上添加动态权重, 增强对分类不准确点的训练, 进一步提升分割的准确度. 在实际工 厂烟尘排放图像数据集上的实验结果表明，所提方法比原有模型在复杂场景下对烟尘的分割结果更准确， $F_{1}$ 度量和 交并比 2 项指标提升显著.
\end{abstract}

关键词：工业烟尘；全卷积网络；多尺度卷积；代价函数

中图法分类号: TP391.41ＤOI: 10.3724/SP.J.1089.2020.18215

\section{Industrial Smoke Target Segmentation Based on Fully Convolutional Networks with Multiscale Convolution and Dynamic Weight Loss Function}

\author{
Liu Hui, Zhang Junpeng ${ }^{*}$, and Li Qingrong \\ (Faculty of Information Engineering and Automation, Kunming University of Science and Technology, Kunming 650500)
}

\begin{abstract}
Smoke blackness is an important indicator in industrial pollution monitoring. Aiming at how to use computer image recognition technology to effectively segment the target area of smoke from the background in the automatic monitoring of Ringelmann scale, and the characteristics of industrial smoke and dust with unfixed shapes and high cloud similarity, leading to the inaccurate results of existing methods to segment smoke in complex scenes. An improved FCN model was proposed, combined multi-scale convolution operations to enhance the feature extraction ability of the network. Dynamic weights were added to the basis of cross entropy to enhance the training of inaccurate points, and the accuracy of segmentation was further improved. The experimental results with the actual factory smoke emission image data set shows that the proposed model is more accurate than the other models in the complex scene, the $F_{1}$-score and the IoU metrics are significantly improved.
\end{abstract}

Key words: industrial smoke; fully convolutional networks; multiscale convolution; loss function

《2015 年环境统计年报》中指出，工业烟尘是 大气污染的主要来源. 对工业烟尘排放进行监测 并及时采取治理措施, 对大气污染治理有着重要
意义. 近年来, 随着图像识别技术的快速发展, 有 学者提出将烟尘图像识别方法用于烟尘排放实时 监测任务中 ${ }^{[1]}$, 其基本思想在于通过分析烟尘图像

收稿日期: 2020-01-17; 修回日期: 2020-10-12. 基金项目：国家自然科学基金(61863018). 刘辉(1984一), 男, 博士, 副教授, 硕 士生导师, CCF 高级会员, 主要研究方向为图像识别; 张俊鹏(1994-), 男, 硕士, 论文通讯作者, 主要研究方向为图像处理与机器视 觉; 李清荣(1990-), 男, 硕士, 主要研究方向为数字图像特征提取与应用. 
特征与林格曼黑度系数之间的对应关系，从而实 现自动、快速地判定当前烟尘污染等级. 基于计算 机视觉的工业烟尘监测任务需要实现包括烟尘图 像采集、烟尘图像分割、烟尘区域特征提取及分析、 特征识别与污染等级判定的功能. 其中, 将烟尘目 标区域从背景图像中准确分割, 是实现烟尘污染 等级图像监测的关键和难点所在.

烟尘图像监测的关键在于实现烟尘图像语义分 割，图像语义分割是指将图像划分为具备不同特征 的区域，并提取出一种或多种感兴趣区域的手段， 它按照类别不同可以划分为传统方法和深度学习方 法 2 大类. 传统的语义分割方法包括 Normalized-cut 方法和 Grab-cut 方法 ${ }^{[2]}$. 其中, Normalized-cut 是一种 基于图划分的方法，其首先对图像建模后，再通过 图理论实现图像的分割; Grab-cut 方法是一种交互 式的方法, 其结合图像的边缘和纹理等特征, 通过 交互操作实现图像分割. 此外, 基于图像灰度直方 图的阈值法 ${ }^{[3]}$ 和基于区域的方法 ${ }^{[4]}$ 也能够实现图像 的分割. 基于深度学习的语义分割方法包括全卷 积网络(fully convolutional networks, FCN ${ }^{[5]}$, Seg$\mathrm{Net}^{[6]}$ 和 U-Net ${ }^{[7]}$ 等深度网络模型. 其中, FCN 通过 替换卷积神经网络的全连接层, 并采用反卷积的 方式实现了图像的像素级的分割; SegNet 通过编 码解码结构的方式增强分割结果的边缘描述; U-Net 采用对称式的结构, 结合浅层的边缘信息和 深层的抽象特征增强图像分割的准确性.

目前，已有大量针对烟尘图像分割问题的研 究, 传统的烟尘分割研究可以分为阈值法 ${ }^{[3-4,8]}$ 、差 分法 ${ }^{[9-10]}$ 和纹理分析法 ${ }^{[1,11-12]}$. 阈值法根据图像的 灰度直方图确定阈值，并根据该阈值划分图像的 每一个像素的类别. 张晓梅等 ${ }^{[4]}$ 将阈值分割法应用 于烟尘图像分割，并使用最大方差法确定分割阈 值. Çelik 等 ${ }^{[3]}$ 采用了颜色建模法检测火灾烟雾，根 据烟尘的灰度和低饱和度特性识别图像中的烟雾 区域. 基于阈值的方法简单且易于实现，但容易将 背景中和烟尘颜色相近的物体判断为烟尘, 且容 易受到天气和光照的影响 ${ }^{[1]}$. 差分法旨在通过对多 幅图像的计算检测其中的运动目标，可以分为帧 间差分法和背景建模法. 魏旭宾等 ${ }^{[10]}$ 首先使用基 于累积序列差分图像的方法计算出大致是烟尘的 区域，之后构造高斯背景统计模型，得出每一个可 能目标点的精确分割阈值，最后进行细分割. 王文 哲等 ${ }^{[13]}$ 提出一种实时更新的背景建模方法, 将当 前图像帧和建立的背景模型进行差分, 以确定图
像中的烟尘区域. 由于差分法检测的是所有移动 的物体，包括飞过的鸟类等干扰物也会被检测出 来，所以抗干扰性不强 ${ }^{[1]}$. 纹理分析法根据烟尘的 纹理特性区分烟尘和背景. Hsu 等 ${ }^{[1]}$ 使用一个包含 5 个向量的滤波器组检测图像中的灰度、边缘、斑 点、波浪以及涟渏，以此确定图像中的烟尘部分. 赵敏等 ${ }^{[14]}$ 提出了基于多种不同纹理特征的烟尘分 割方法，在背景建模阶段综合考虑了图像的空间 以及时间特性，以确定大致的烟尘区域; 之后提取 烟尘区域的 3 种纹理特征, 并训练支持向量机用 于分类. 而纹理分析法过于依赖人工设计的特征， 以致方法的适应性不强，应用于不同场景时表现 不佳 ${ }^{[15]}$.

目前，深度学习被广泛应用于图像分类 ${ }^{[16]}$ 、目 标检测 ${ }^{[17]}$ 等任务中, 相比于传统数字图像处理的 方法, 其使用大量数据训练网络模型, 所以在适应 性方面表现更佳, 它应用于烟尘图像分割任务中 也取得了相比于传统方法更佳的结果. 邓林 ${ }^{[18]}$ 提 出使用反向传播(back propagation, BP)神经网络进 行视频中的烟雾检测, 它可以有效地检测视频中 的烟雾, 同时具备一定的抗干扰能力; 但是在干扰 较多、场景变化较大的情况下，检测效果会有所下 降. 王文哲 ${ }^{[19]}$ 使用 FCN 对烟尘图像进行分割, 首 先人工标记图像中的烟尘区域制作数据集，再对 $\mathrm{FCN}$ 进行训练, 最终得到像素级的烟尘分割结果. 但该方法容易受到背景中物体的干扰，在复杂场 景下的分割准确度仍然有待提高. 因此, 具有较好 环境适应能力的烟尘图像分割算法仍需要进一步 研究和分析.

由于 FCN 在图像语义分割中能够实现像素级 的图像分割, 本文以该网络模型作为基础模型框 架，针对使用 FCN 进行烟尘图像分割时存在的易 受干扰导致分割不准确的问题, 融人了多尺度卷 积元素, 并改进了代价函数, 从而构建了一种采用 多尺度卷积操作的 FCN. 其特点在于克服了单一 尺寸卷积核的特征提取能力有限的问题, 通过多 个不同尺度的卷积核会产生多个尺度的感受野, 多尺度特征的融合会加强网络的特征提取能力. 相比于原有的交叉熵代价函数，提出的结合动态 权重的代价函数可以增强对分类不准确点的训练, 同时减少对分类准确点的训练. 通过提高网络模 型的训练效率，进一步提升模型分割烟尘的准确 度. 在多个场景下的测试结果表明，本文方法可以 更准确地分割出烟尘区域. 


\section{FCN}

Shelhamer 等 ${ }^{[5]}$ 提出的 FCN 是最经典的用于图 像语义分割的网络模型, 它是一种端到端的网络, 其输人可以是任意大小的图像，经过网络的计算 并对每一个像素进行分类, 生成和原始图像尺寸 一致的像素级分割结果.

\section{1 卷积、池化与反卷积}

$\mathrm{FCN}$ 根据用于图像分类的卷积神经网络改造 而成, 网络的前半部分是卷积神经网络堆叠的卷 积、池化和激活函数操作 ${ }^{[20]}$. 其中卷积操作用于图 像的特征提取, 激活函数用于增加网络的非线性, 池化操作旨在减少计算量的同时增加网络的感受 野. 图像经过这一过程可以表示为

$$
x_{m+1}=R\left(P\left(w * x_{m}+b\right)\right)
$$

其中， $x_{m}$ 指卷积层的输人图像; $w$ 指卷积核的权 重; $*$ 表示卷积操作; $b$ 表示偏置; $P$ 表示池化操 作; $R$ 表示激活函数; $x_{m+1}$ 表示输出结果.

网络的后半部分将卷积神经网络中用于将特 征映射到分类的全连接层替换为卷积层, 通过这 一操作保留了特征图的空间信息, 对最后一层卷 积层输出的特征图进行反卷积操作将其还原至原 始图像尺寸. 反卷积 ${ }^{[21]}$ 又称为转置卷积, 是一种 特殊的正向卷积. 反卷积的操作首先在特征图的 周围填充 0 , 旋转卷积核后再进行正向卷积. 经过 反卷积操作将特征图还原到原始图像的尺寸, 再 通过对每个像素分别进行预测, 实现对图像的像 素级分类.

\section{2 跳跃结构}

网络中多次池化层操作虽然增大了感受野, 但代价是特征图的尺寸越来越小; 最后一层卷积 层输出的特征图的尺寸是原始图像的 $1 / 32$. 为将 输出结果还原到原始图像的尺寸, 通过反卷积的 方式对其进行 32 倍上采样. 虽然还原到和原始图 像同样的尺寸，但由于池化过程中丢失了大量的 空间信息，会导致结果较为模糊. 针对此问题, FCN 通过跳跃结构融合深层和浅层的特征信息， 由此产生了 FCN-16s 和 FCN-8s 2 种衍生模型. FCN-16s 模型对最后一层卷积层输出的特征图进 行 2 倍上采样后与第 4 个池化层的特征图相加, 之 后对融合的结果进行 16 倍上采样生成最终的预测 图. 在此基础上, $\mathrm{FCN}-8 \mathrm{~s}$ 结合了第 3 层池化层的特 征图信息对分割结果进行进一步的细化.

\section{2 结合多尺度卷积的 $\mathbf{F C N}$}

工业烟尘是一种非刚体物质，它不具备固定 的形状以及清晰的边缘. 在排放过程中, 形状时大 时小, 而且灰度变化较大, 有时呈现出透明性, 颜 色也常发生变化. 现有的分类网络大多是针对日 常的固定形状的物体进行分类, 工业烟尘的这些 特有的属性导致 FCN 应用于烟尘图像分割任务中 表现不佳, 对烟尘的分割性能还有待提高. 本文通 过在 FCN 的基础上加人多尺度卷积结构, 来增强 网络对工业烟尘的分割效果.

\section{1 多尺度卷积结构}

在 GoogLeNet ${ }^{[22]}$ 之前, 为了提高神经网络的 性能, 主要采用增加网络深度的做法, 即在网络中 设置更多的卷积层. 这种实现方式虽然可以达到 目的，但也带来了其他的隐患. 首先，网络的加深 伴随着参数量的提升, 在训练集数据不足的情况 下, 更容易出现过拟合的问题; 其次, 更多层意味 着计算复杂度的提高, 训练过程中容易出现梯度 弥散的问题, 使得网络难以优化.

Inception ${ }^{[23]}$ 结构的思想是构建密集的块结 构，使用不同尺寸的卷积核分别对特征图进行卷 积运算, 将几组特征图融合后再进行下一层的运 算, 融合方式是将几组经不同卷积处理的特征图 在表示通道数目的维度上进行拼接. 这在增加网 络宽度的同时, 增加了对尺度的适应性. Inception 结构的提出, 使得在计算量不显著增加的前提下 增加网络的宽度和深度成为可能, 其模块结构如 图 1 所示.

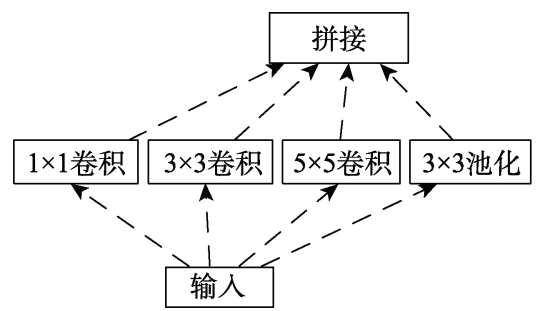

图 1 Inception 模块结构

在工业烟尘排放过程中, 从图像识别的角度 分析存在以下的难点:

(1) 烟尘在排放过程中其大小变化不稳定, 在 不同排放阶段其面积具有一定的随机性, 特别是 当烟尘在初始排放阶段会出现缩小的情况, 而加 深的深度网络对此种烟尘检测并不敏感.

(2) 实际烟尘排放过程中, 有可能存在多个排 
放点，具有不同的特征且面积大小不固定，因此单 一尺度下的网络模型对此类目标检测难以适应.

Inception 结构通过增加网络宽度提高网络的 性能，避免了过深的网络难以捕获小目标烟尘的 问题; 通过在同一层设置不同尺寸的卷积核提取 不同的烟尘特征，不同尺度的卷积核对于不同大 小的烟尘具有更好的适应性，在多尺度卷积后进行 拼接以合成特征，从而感知不同尺度的烟尘特征.

\section{2 多尺度卷积 $\mathrm{FCN}$}

本文网络结构如图 2 所示, 输人层接收任意尺 寸的 3 通道烟尘图像, 在此以输人图像尺寸是 $224 \times 224$ 像素为例进行说明. 网络的前端是多层交 替的卷积层和池化层, 包括 5 层卷积层和 4 层平均 池化层, 第 5 层池化层的池化计算方式为最大池 化. 网络各层的参数设置如表 1 所示, 其中过滤器 和输出的尺寸的单位是像素 $\times$ 像素 $\times$ 通道数目.

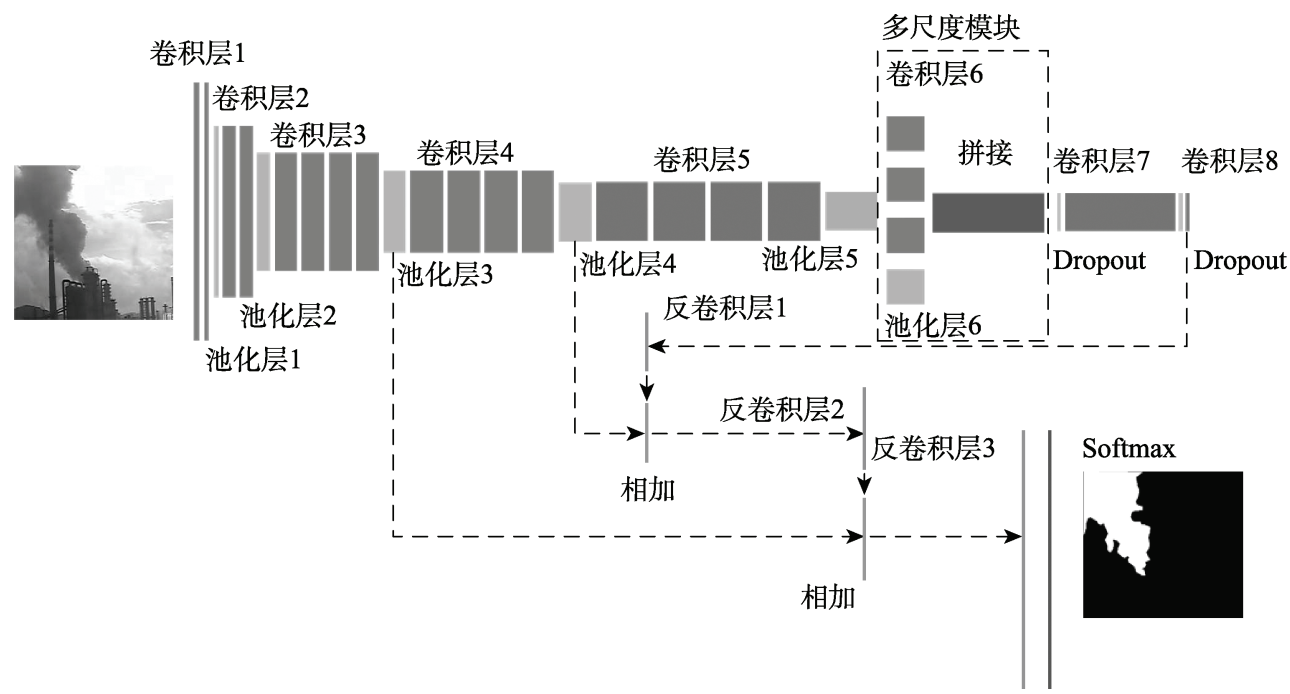

图 2 本文网络结构

表 1 本文网络参数设置

\begin{tabular}{cll}
\hline \multicolumn{1}{c}{ 层 } & 过滤器尺寸 & 输出尺寸 \\
\hline 卷积层 1 & $3 \times 3 \times 64$ & $224 \times 224 \times 64$ \\
池化层 1 & $2 \times 2$ & $112 \times 112 \times 128$ \\
卷积层 2 & $3 \times 3 \times 128$ & $112 \times 112 \times 128$ \\
池化层 2 & $2 \times 2$ & $56 \times 56 \times 128$ \\
卷积层 3 & $3 \times 3 \times 256$ & $56 \times 56 \times 256$ \\
池化层 3 & $2 \times 2$ & $28 \times 28 \times 256$ \\
卷积层 4 & $3 \times 3 \times 512$ & $28 \times 28 \times 512$ \\
池化层 4 & $2 \times 2$ & $14 \times 14 \times 512$ \\
卷积层 5 & $3 \times 3 \times 512$ & $14 \times 14 \times 512$ \\
池化层 5 & $2 \times 2$ & $7 \times 7 \times 512$ \\
卷积层 6_1 & $1 \times 1 \times 128$ & $7 \times 7 \times 128$ \\
卷积层 6_2 & $3 \times 3 \times 128$ & $7 \times 7 \times 128$ \\
卷积层 6_3 & $7 \times 7 \times 128$ & $7 \times 7 \times 128$ \\
池化层 6 & $3 \times 3$ & $7 \times 7 \times 128$ \\
卷积层 7 & $1 \times 1 \times 4096$ & $7 \times 7 \times 4096$ \\
卷积层 8 & $1 \times 1 \times 512$ & $7 \times 7 \times 512$ \\
反卷积层 1 & $4 \times 4 \times 512$ & $14 \times 14 \times 512$ \\
反卷积层 2 & $4 \times 4 \times 256$ & $28 \times 28 \times 256$ \\
反卷积层 3 & $16 \times 16 \times 2$ & $224 \times 224 \times 2$ \\
\hline
\end{tabular}

文献[24]中指出，考虑计算量的问题，在浅层 保持传统卷积结构, 而在深层开始使用 Inception 结构也是较为有效的, 所以保留 FCN 前 5 层卷积
层的参数设置, 只对较深的卷积层进行改造. 对原 本使用了尺寸为 $7 \times 7$ 卷积核的第 6 层卷积层参照 Inception 结构进行替换, 如图 3 所示.

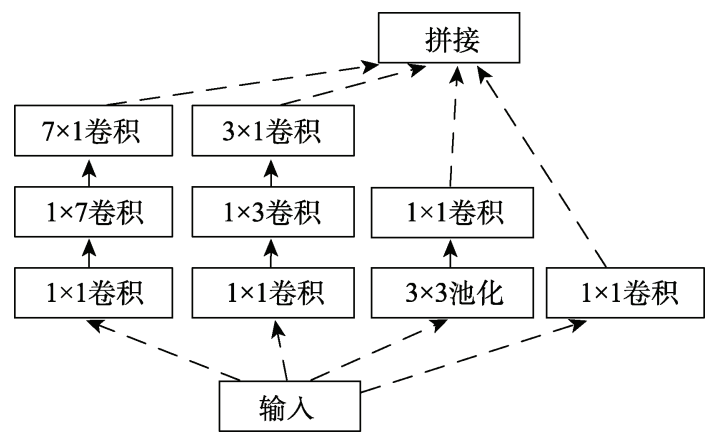

图 3 多尺度卷积结构

在这一层使用 3 种不同尺寸 $1 \times 1,3 \times 3,7 \times 7$ 的 卷积核替换掉原有的单一的卷积核, 通过这一操 作引人多尺度感受野. 为减少计算量, 在较大的 2 个卷积核前添加 $1 \times 1$ 大小的卷积核, 用于减少特 征图的通道数目, 这一系列的运算可以表示为

$$
\begin{aligned}
& y_{1}=w_{1} * x_{m}+b_{1} \\
& y_{2}=w_{22} *\left(w_{21} * x_{m}+b_{21}\right)+b_{22}
\end{aligned}
$$




$$
\begin{aligned}
& y_{3}=w_{32} *\left(w_{31} * x_{m}+b_{31}\right)+b_{32} \\
& y_{4}=w_{4} * f_{\text {pool }}\left(x_{m}\right)+b_{4} \\
& x_{m+1}=f_{\text {concat }}\left(y_{1}, y_{2}, y_{3}, y_{4}\right)
\end{aligned}
$$

其中， $x_{m}$ 表示这一层的输人特征图; $w_{i}$ 和 $b_{i}$ 分别 表示每一分支的卷积操作的过滤器和偏置; * 表 示卷积操作; $f_{\text {pool }}$ 表示池化操作; $f_{\text {concat }}$ 表示将 4 组 特征图 $y_{1} \sim y_{4}$ 在第 3 维度上进行拼接; $x_{m+1}$ 表示输 出的结果.

同时将 2 个较大尺寸的卷积核 $3 \times 3$ 和 $7 \times 7$ 拆 分为 2 个串联的一维卷积核 $(1 \times 3,3 \times 1 ; 1 \times 7,7 \times 1)$, 使用较小的非对称卷积核替换较大的卷积核的优 势是：在实现同样感受野的基础上有效地减少计 算量，同时增加了网络的深度和非线性 ${ }^{[23]}$. 考虑 池化层对现有的卷积网络的重要性，添加第 4 个分 支用于池化计算. 因为图像经过不同尺寸过滤器 计算后图像的尺寸也不同，所以在这一层使用特 征图周围填充 0 的方式，保持 4 个分支的输出结果 具有一致的尺寸 $(7,7 ， 128)$, 其中前 2 个维度表示 特征图的尺寸, 第 3 维度表示特征图的通道数. 在 第 3 个维度上进行拼接后作为下一层卷积层的输 人. 经过最后一层卷积层计算输出的热度图尺寸 是原始图像的 $1 / 32$. 为了获得更精细的分割结果, 使用跳跃结构融合浅层的特征图信息：对热度图 进行 2 倍上采样恢复至第 4 层池化特征图的尺寸, 融合后再进行一次 2 倍上采样，与第 3 层池化后的 特征图融合, 最后进行一次 8 倍上采样还原至输人 图像尺寸, 形状为 $(224,224,2)$, 第 3 维度的 2 表示 分类数目. 经过 Softmax 的归一化处理, 将数值转 换为概率分布. 某一位置在第 3 维度有 2 个概率值, 分别表示为背景的预测概率和烟尘的预测概率. 通过比较 2 个概率值的大小, 确定该位置的像素所 属类别, 得到最终的预测图.

\section{3 融合动态权重的交叉熵代价函数}

在前期工作中，使用 FCN 对训练集进行了 20 轮迭代，但生成的模型在诸如背景中存在云干扰 的测试集上表现不佳，常受到背景中云的干扰以 至于分割结果中存在错误分类点. 分析原因是在 训练网络过程中没有对错误分类点进行有效的训 练，以下对该情况进行具体分析.

\subsection{1 交叉熵函数的局限性分析}

模型的训练旨在随着每次迭代最小化网络的 损失，以图 4 为例对损失的计算进行说明.

对热度图进行 Softmax 归一化为概率分布，可 以表示为图 4 中热度图到预测结果的过程. 图 4 预

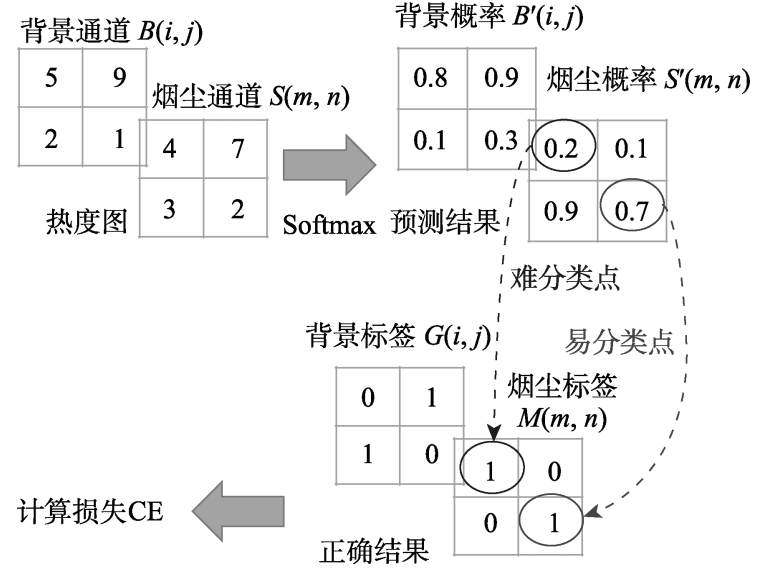

图 4 网络损失计算

测结果中的 $B^{\prime}(i, j)$ 表示预测为背景的概率, $S^{\prime}(m, n)$ 表示预测为烟尘的概率. 通过对第 3 维度 上 2 个概率的比较从而确定其类别, 因为 $B^{\prime}(1,1)$ 和 $B^{\prime}(1,2)$ 位置表示被预测是背景的概率分别为 0.8 和 0.9 , 高于是烟尘的概率 0.2 和 0.1 , 所以将此 2 点判断是背景; $S^{\prime}(2,1)$ 和 $S^{\prime}(2,2)$ 位置被预测是烟 尘的概率分别为 0.9 和 0.7 , 高于被预测是背景的 概率 0.1 和 0.3 , 所以预测该 2 点为烟尘. 图 4 的正 确结果中, $G(i, j)$ 表示背景标签, $M(m, n)$ 表示烟 尘标签. 其中, $M(1,1)$ 和 $M(2,2)$ 位置是烟尘, $M(1,2)$ 和 $M(2,1)$ 是背景. 因为 $M(1,1)$ 位置是烟 尘, 所以被预测是烟尘的概率应尽量接近 1 , 但由 于被网络预测是烟尘的概率为 0.2 , 认定其为分类 不准确的点, 也是错误分类点; $M(2,2)$ 位置也是 烟尘, 而该位置被预测是烟尘的概率为 0.7 , 认定 其为分类较为准确的点.

根据图 4 中预测结果与正确结果计算得出网 络的损失. 语义分割网络常用的损失函数是交叉熵 (cross entropy, $\mathrm{CE})^{[25]}$ 二 二分类条件下的 $\mathrm{CE}$ 表示为

$$
\begin{gathered}
\mathrm{CE}=-y \ln \hat{y}-(1-y) \ln (1-\hat{y})= \\
\begin{cases}-\ln \hat{y}, & y=1 \\
-\ln (1-\hat{y}), & y=0\end{cases}
\end{gathered}
$$

其中, $y=1$ 表示正样本, $y=0$ 表示负样本; $\hat{y}$ 为 网络预测结果, 是 $0 \sim 1$ 的概率值.

对各样本点的损失求和得到总体的损失，再 进行反向传播, 通过梯度下降策略调整网络中的 各项权值. 使用标准交叉熵代价函数局限之处在 于, 随着训练的进行, 除了少数的难分类的样本 点, 越来越多的样本点成为分类正确的样本, 因此 会出现以下的 2 种情况:

（1）对于易分类、分类正确的单个样本点, 其 
概率更接近标签值，所以通过交叉熵函数计算得 出的损失较小. 虽然每一样本点的误差较小, 但因 为占据了总体中的大多数, 所以在总体损失中也 会占据较大的比例.

(2) 而对于难分类、分类错误的样本点，其概 率与标签值相差较大，所以通过交叉熵计算得到 的损失也较大. 虽然每一样本点的误差较大, 但是 数量较少, 因此在总体损失中所占比例也较小.

大量的易分类样本点会引导梯度下降的方向， 以至于对难分类样本点训练不充分.

\subsection{2 动态加权的交叉熵函数}

本文提出的融合动态权重代价函数的思想是 更关注难分类、错误分类点，减少对易分类、正确 分类点的关注度. 提高分类不准确的点在总体损 失中所占比例，以便于在反向传播阶段对权重进 行更多的调节; 同时降低分类准确点在总体损失 中所占的比例, 减弱对权重的调节. 实现方式是采 用一个变换后的 Sigmoid 函数对该点的概率进行 $0 \sim 1$ 内的映射, 并将此映射值作为权重添加在交叉 熵之前, 以动态地调整损失值在总体的损失中所 占的比例.

Sigmoid 函数 ${ }^{[26]}$ 常被用作神经网络中的激活 函数, 用于将变量映射至 $0 \sim 1$, 表示为

$$
S(x)=\frac{1}{1+\mathrm{e}^{-x}}
$$

其对应的函数曲线如图 5 黑色曲线所示. 因为要对 $0 \sim 1$ 的概率值进行映射, 所以将 Sigmoid 函数进行 平移、缩放与翻转调整至如图 5 所示的灰色曲线, 变换后的公式表示为

$$
S^{\prime}(x)=\frac{1}{1+\mathrm{e}^{10 x-5}}
$$

最终的代价函数设计为

$$
L= \begin{cases}-S^{\prime}(\hat{y}) \ln \hat{y}, & y=1 \\ -S^{\prime}(1-\hat{y}) \ln (1-\hat{y}), & y=0\end{cases}
$$

其中， $\hat{y}$ 表示预测是烟尘的概率; $y=1$ 表示烟尘 分类； $y=0$ 表示背景分类.

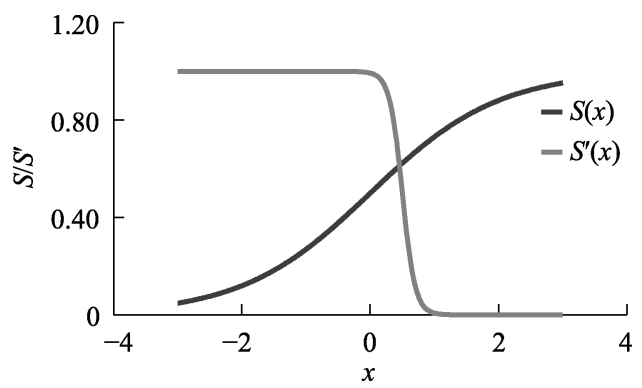

图 5 变换的 Sigmoid 函数曲线
对于是烟尘但被预测是烟尘的概率为 0.2 的难 分类点, 经过式(10)所示函数映射得到的权重为 0.953; 对于被预测是烟尘概率为 0.9 的易分类烟尘 点, 经过式(10)函数的映射结果为 0.018 . 根据每 一位置上与正确值(即 1)的偏差程度调整交叉熵前 权重的大小，放大总体中难分类点的损失占比，减 少易分类点的损失占比，以调整其在误差反向传 播阶段进行权值调整的幅度, 通过这种方式增强 对分类不准确点的训练, 进一步提升模型分割烟 尘的准确度.

\section{3 实验与分析}

\section{1 实验平台及数据}

网络的训练在配备 Intel(R) Xeon(R) CPU E5-2620v4@2.10 GHz 处理器, 64 GB 内存, NVIDIA GeForce RTX 2080Ti 显卡的工作站上进 行, 使用的深度学习平台是 TensorFlow. 训练网络 所用数据来自于实际钢厂定点拍摄的工业烟尘排 放视频，视频记录了白天时间段内不同监测点以 及不同场景的烟尘排放信息. 共选取了 60 段视频, 提取 1289 幅烟尘排放图像, 使用 Labelme 对图像 中的烟尘区域进行手动标记作为标签. 对图像及 其对应标签进行 $90^{\circ}, 180^{\circ}, 270^{\circ}$ 旋转和上下翻转操 作, 扩充数据集至 6445 幅. 其中训练集图像为 5260 幅, 测试集图像为 1185 幅. 图像的尺寸统一 设置为 $224 \times 224$ 像素. 为验证本文方法对不同场 景下烟尘的分割效果, 将测试集图像分为图 6 所示 的 5 个场景, 其中第 1 行为原始图像, 第 2 行为对 应的标签图. 各个场景下测试图片数量如表 2 所示.

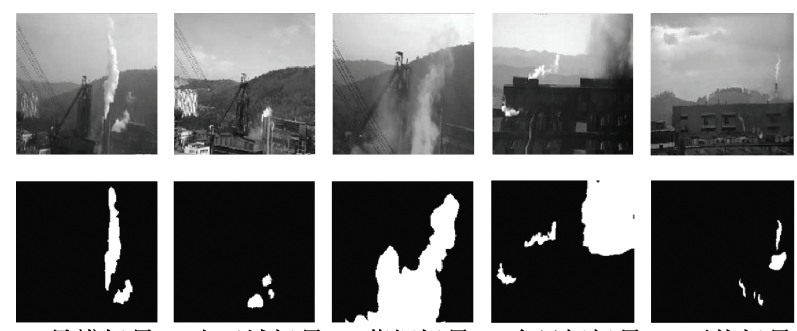

a. 易辨场景 b. 小区域场景 c. 薄烟场景 d. 多目标场景 e. 干扰场景

图 6 场景分类示意

表 2 场景分类数目

\begin{tabular}{lcc}
\hline \multicolumn{1}{c}{ 场景分类 } & 测试集数量 & 训练集数量 \\
\hline 易辨场景 & 339 & \\
薄烟场景 & 173 & \\
多目标烟尘场景 & 303 & 5260 \\
小区域烟尘场景 & 160 & \\
干扰(云)场景 & 210 & \\
\hline
\end{tabular}




\section{2 评价指标}

为了定量分析工业烟尘的分割结果, 选择了 查准率 $P$ 、查全率 $R, F$ 度量 ${ }^{[27]}$ 与交并比(intersection over union, IoU $)^{[4]}$ 作为评价指标. 查准率是被正确 分类的正样本数量与被预测为正样本的数量之比, 定义为

$$
P=\frac{p_{i i}}{P_{i i}+P_{j i}}
$$

查全率是被正确分类的正样本数量与正样本数量 之比，定义为

$$
R=\frac{p_{i i}}{p_{i i}+p_{i j}}
$$

因为查全率和查准率二者表现出一定的矛盾性, 其中一项指标较高时, 另一项往往较低, 所以通过 $F$ 度量指标综合考量这 2 项指标，同时参考查准率 和查全率的度量, 其定义为

$$
F=\frac{\left(1+\beta^{2}\right) \times P \times R}{\left(\beta^{2} \times P\right)+R}
$$

其中, $\beta$ 用于调整权重, 若认为查准率重要, 则减 小 $\beta$, 若认为查全率重要, 则增大 $\beta ; \beta=1$ 时认 为二者一样重要, 设置 $\beta=1$, 以综合评价查全率 和查准率指标. IoU 是图像语义分割的标准度量, 是真实值和预测值的比值, 定义为

$$
\mathrm{IoU}=\frac{p_{i i}}{p_{i i}+p_{i j}+p_{j i}}
$$

其中，在实验中有 2 个分类：用 $i$ 表示烟尘(正样 本), 用 $j$ 表示背景(负样本); $p_{i i}$ 指属于类别 $i$ 被 预测为 $i$ 的像素数量; $p_{i j}$ 指属于类别 $i$ 但被预测 为 $j$ 的像素数量; $p_{j i}$ 指属于类别 $j$ 但被预测为 $i$ 的像素数量.

\section{3 模型结构对比}

为了验证多尺度卷积与动态权重代价函数对 烟尘分割准确性方面的提升，本节将原始的 FCN 模型、添加多尺度卷积的 FCN 模型(记作 $\mathrm{mFCN}$ ), 以及添加动态权重代价函数的模型(记作 $\mathrm{mFCN}-\mathrm{s}$ ) 进行了对比. 训练中设置批量为 1 , 学习率 $=0.0001$, 对训练集图像迭代 100000 次后, 对比 3 种模型在 测试集上的分割结果与量化指标. 量化指标对比 结果如表 3 所示, 分割结果对比如图 7 所示.

\begin{tabular}{lcccc}
\multicolumn{1}{c}{ 表 3} & \multicolumn{2}{c}{ 不同模型在测试集上量化指标对比 } & \multicolumn{1}{c}{$\%$} \\
\hline \multicolumn{1}{c}{ 模型 } & IoU & $F_{1}$ & $P$ & $R$ \\
\hline $\mathrm{FCN}^{[5]}$ & 77.79 & 87.60 & 85.20 & 90.14 \\
$\mathrm{mFCN} / \mathrm{mFCN}-\mathrm{ce}$ & 78.76 & 88.27 & 89.15 & 87.40 \\
$\mathrm{mFCN}-$ focal $^{[25]}$ & 78.49 & 87.98 & 85.71 & 90.38 \\
$\mathrm{mFCN}-\mathrm{s}$ & 80.06 & 89.01 & 90.85 & 87.24 \\
\hline
\end{tabular}
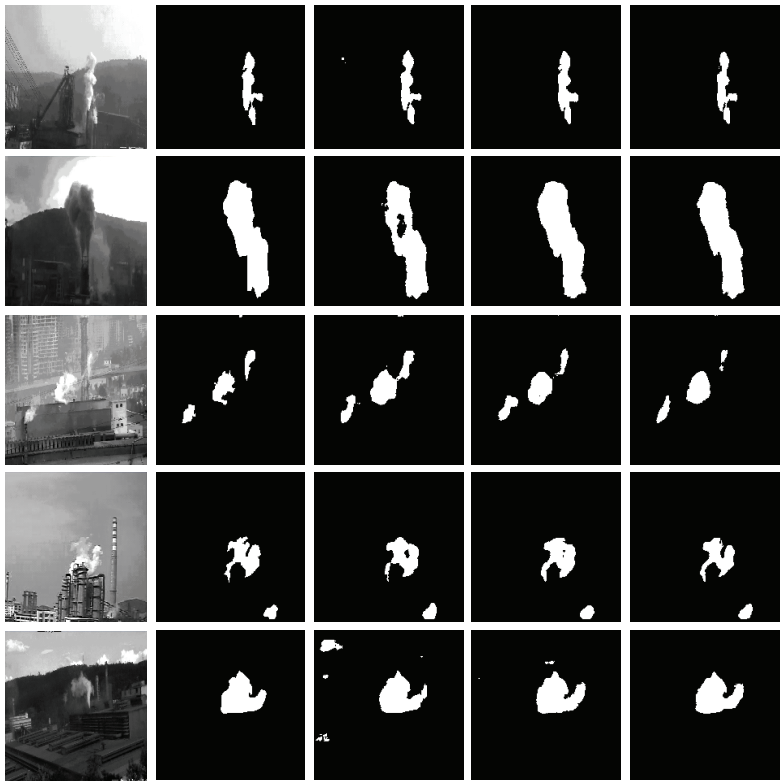

a. 原图 b. 人工标记

d. $\mathrm{mFCN}$

e. $\mathrm{mFCN}-\mathrm{s}$

图 7 不同模型分割结果对比

图 7 分割结果表明, 对于 FCN 分割结果中存 在的空洞(如图 7c 第 2 行), 以及受到背景干扰的问 题(如图 7c 第 1 行和第 5 行), $\mathrm{mFCN}$ 有所改善, 因 为多尺度卷积对于不同特征烟尘的提取能力更强, 但仍然存在受干扰(如图 7d 第 5 行) 以及边缘不准 确的问题 (如图 $7 \mathrm{~d}$ 第 1 行和第 4 行). 为代价函数添 加动态权重后, 对于受干扰问题有了进一步的改 善(如图 7e 第 5 行), 边缘也更加准确(如图 $7 \mathrm{e}$ 第 1 行和第 4 行), 这是因为对于带有动态权重的代价 函数在训练过程中对难分类的边缘和干扰区域进 行了更有效的训练. 在量化指标方面, 添加多尺度 和动态权重代价函数后的 IoU 以及 $F_{1}$ 度量 2 项指 标比改进前均有提升.

\section{4 代价函数对比}

为了验证本文代价函数的有效性，在多尺度 卷积 FCN 结构的基础上, 对比了采用交叉熵(记作 $\mathrm{mFCN}$-ce), focal loss ${ }^{[25]}$ (记作 $\mathrm{mFCN}$-focal), 以及本 文代价函数(记作 $\mathrm{mFCN}-\mathrm{s}$ ) 3 种模型的分割结果, 如 图 8 所示, 量化指标对比结果如表 3 所示.

图 8 分割结果表明，采用交叉熵的模型易出现 分割结果不完整的问题(如图 $8 \mathrm{c}$ 第 2 行、第 4 行和 第 5 行), 因为训练过程中交叉熵代价函数没能够 对分类不准确目标进行有效训练. 采用 focal loss 的模型的分割结果比交叉熵模型分割结果更完整, 但仍然存在边缘不准确的问题(如图 $8 \mathrm{~d}$ 第 1 行). $\mathrm{mFCN}-\mathrm{s}$ 的动态加权因子加强了对分类不准确样本 


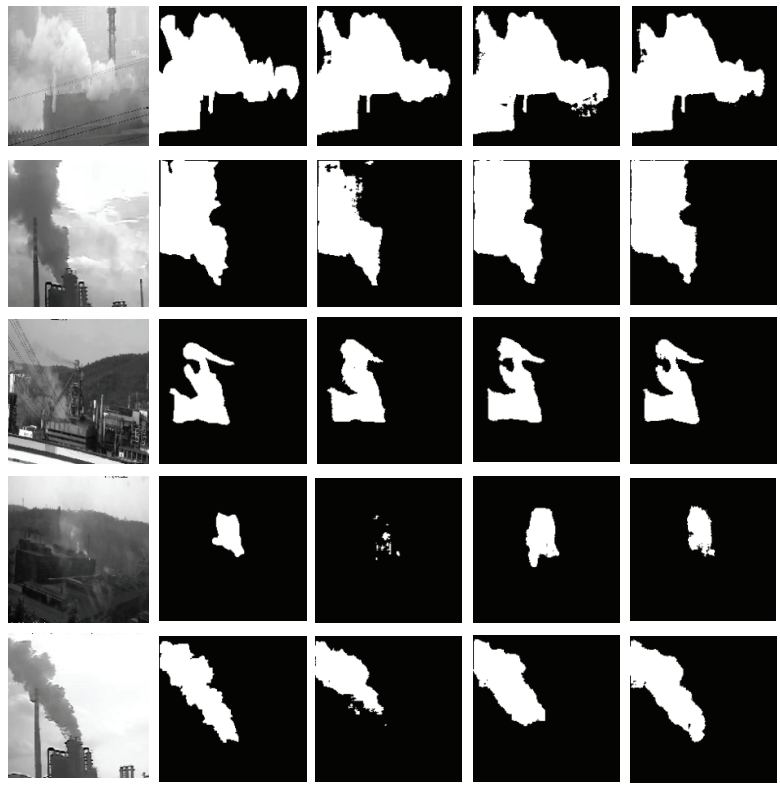

a. 原图 b. 人工标记 c. mFCN-ce d. mFCN-focal ${ }^{[2]}$ e. $m F C N-s$

图 8 采用不同代价函数的分割结果对比

点的训练, 所以在分割结果的完整性以及边缘准 确性方面更加准确. 量化指标对比结果同样表明, 在 $\mathrm{IoU}$ 和 $F_{1}$ 度量 2 项指标上, 本文 $\mathrm{mFCN}-\mathrm{s}$ 模型 的烟尘分割准确度均优于其他代价函数模型.

\section{5 与其他模型的对比}

本节对本文的模型同其他 4 种深度网络模型 进行了对比, 包括的 FCN 模型 ${ }^{[19]}$ (记作 FCN), 文 献[6]方法(记作 en-de), 文献[7]中的方法(记作 u-net), deeplabv3+方法 ${ }^{[28]}$ (记作 deeplab). 其中，标 准 deeplabv3+的主干网络部分是 101 层或 50 层的 卷积结构, 考虑实验数据量以及对比模型的规模,
对主干网络的层数进行了调整, 使用 16 层的 Resnet 网络, 下采样次数和原论文模型保持一致, 为 5 次下采样. 训练过程中的超参数均设置为批量 $=1$, 学习率 $=0.0001$, 迭代次数 $=100000$. 之后在 5 个场 景的测试集上进行测试, 分割结果对比分别如图 9 图 13 所示, 测试结果量化指标对比分别如表 4 表 7 所示.

（1）在图 9 易辨场景对比中, en-de 和 u-net 的 分割结果中存在空洞(图 9c 第 1 行、图 9d 第 1 行), $\mathrm{FCN}$ 的结果中包含小部分的非烟尘区域(如图 9f 第 3 行和第 4 行), deeplab 和 $\mathrm{mFCN}-\mathrm{s}$ 的分割结果更准确.

(2) 在图 10 小区域场景对比中, en-de 没能识 别出小区域烟尘(图 10c 第 4行), u-net 和 FCN 错误 识别小部分背景区域(图 10d 第 3 行、图 10f 第 3 行), 而 deeplab 和 mFCN-s 的分割结果更准确.

（3）在图 11 薄烟场景对比中, en-de 和 deeplab 结果的边缘不准确(图 11c第3,4行, 图 11e第3行), u-net 分割的区域不完整(图 11d 第 3 行), mFCN-s 在边缘和分割完整性方面表现更佳.

（4）在图 12 多目标场景对比中, en-de, u-net, deeplab, FCN 均没能完整识别颜色较深的烟尘区 域(图 12 第 2 行), en-de 的边缘较不准确, mFCN-s 采用的多尺度卷积对于多种特征的烟尘识别较为 准确(图 12g 第 2 行).

(5) 图 13 干扰场景对比中, en-de 分割结果不 完整(图 13c 第 3 行和第 4 行), u-net 和 deeplab 结 果中有空洞(图 13d 第 4 行、图 13e 第 4 行), u-net 没能识别边缘部分的烟尘(图 13d 第 2 行), FCN 受

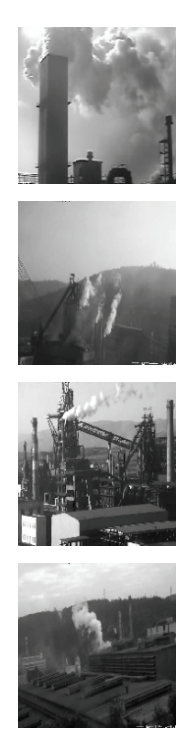

a. 原图
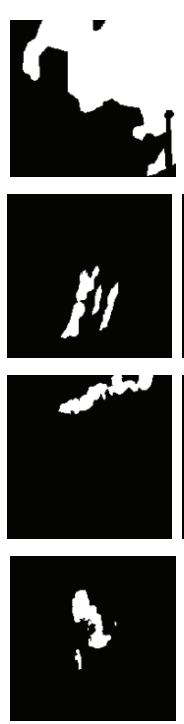

b. 人工标记

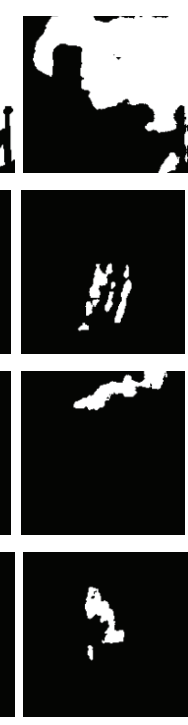

图 9 易辨场景分割结果对比

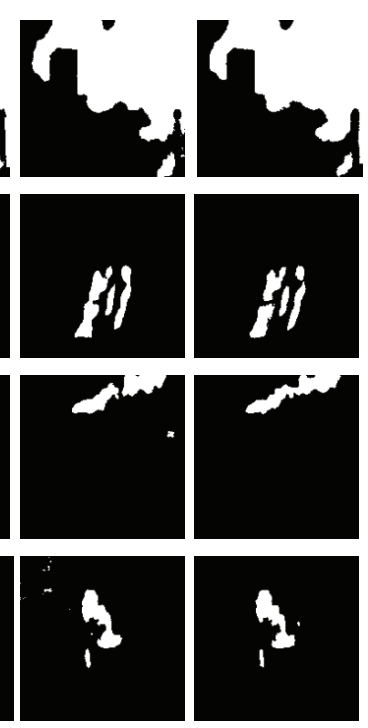

g. $\mathrm{mFCN}-\mathrm{s}$

f. $\mathrm{FCN}^{[5]}$ 


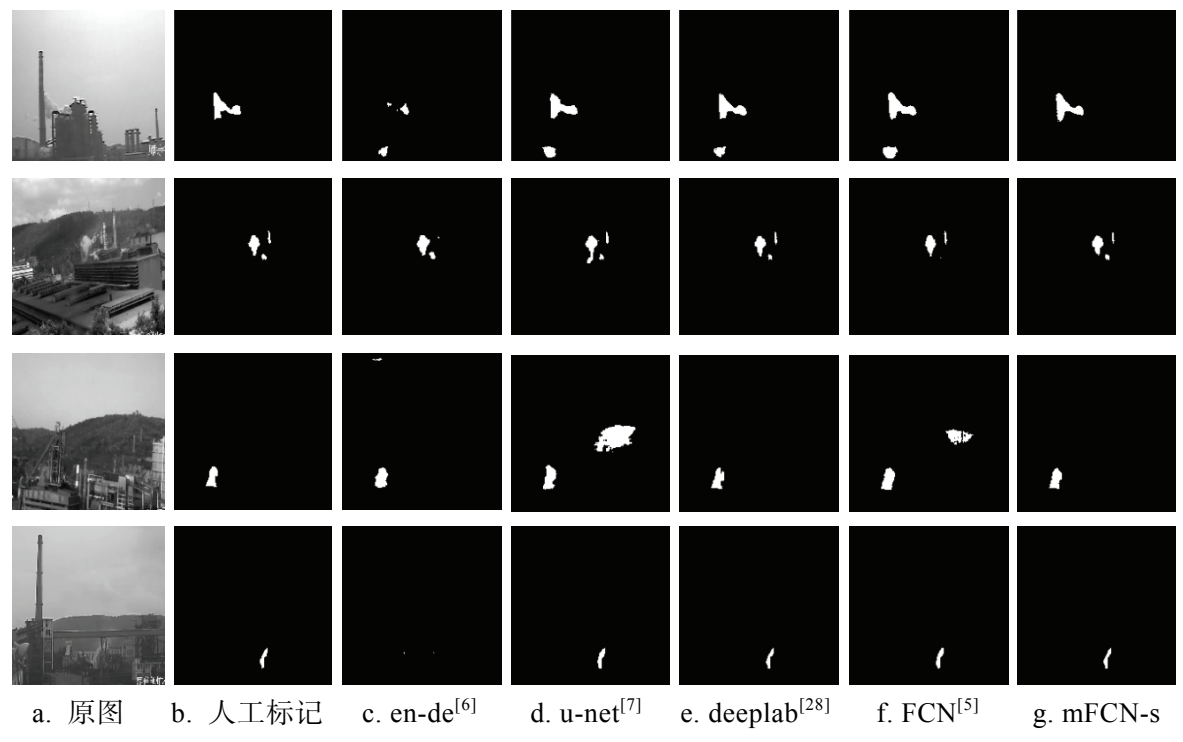

图 10 小区域场景分割结果对比

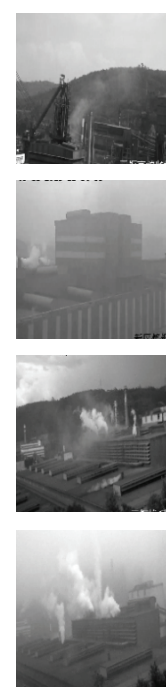

a. 原图
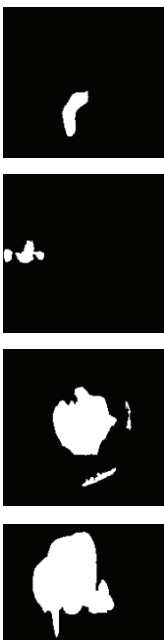

b. 人工标记
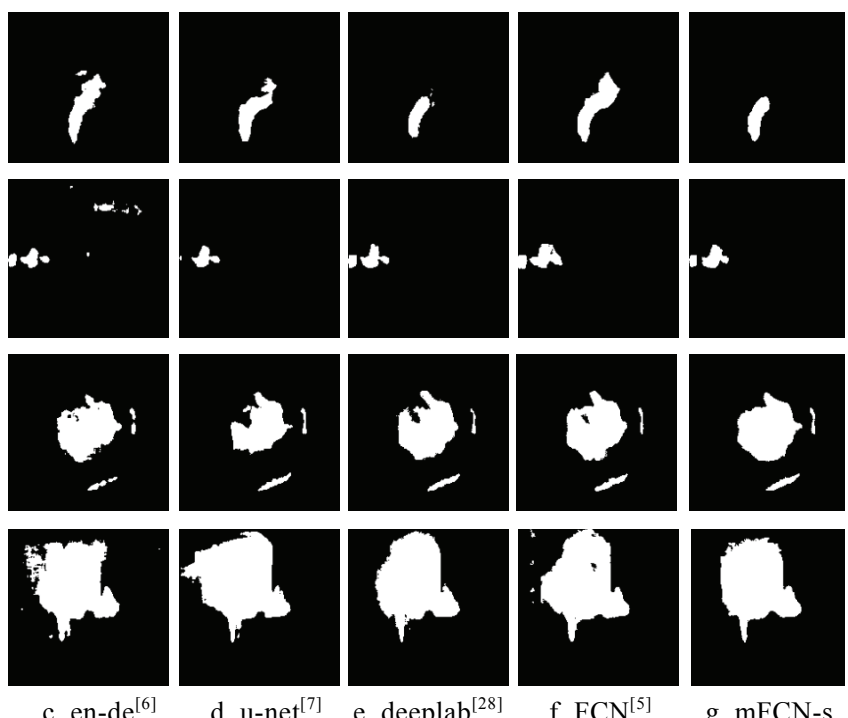

图 11 薄烟场景分割结果对比

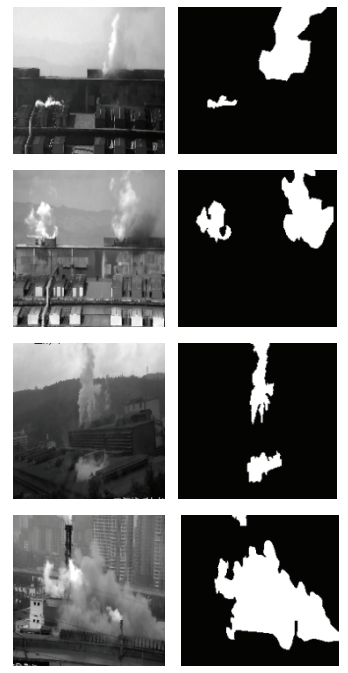

a. 原图
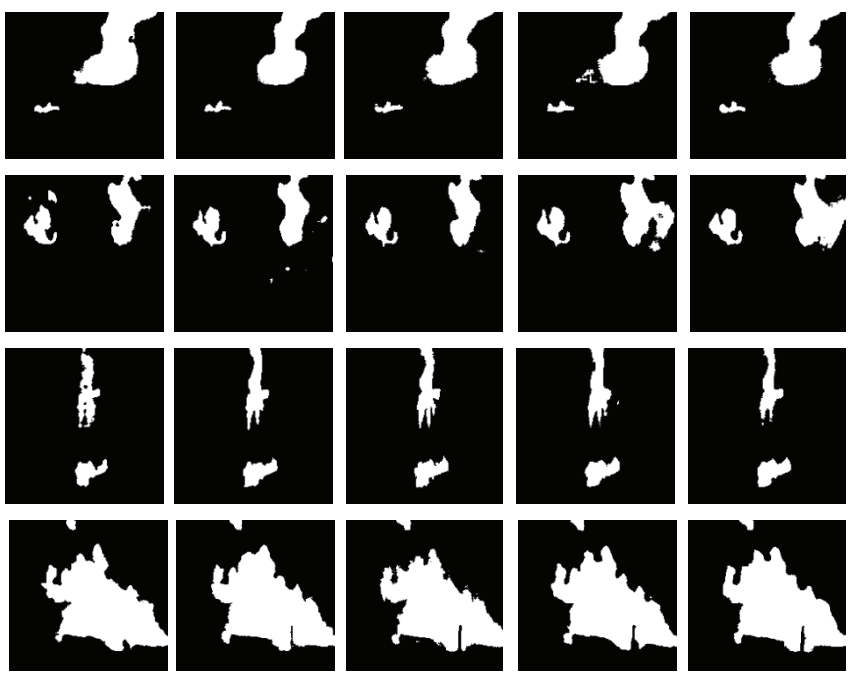

图 12 多目标场景分割结果对比 


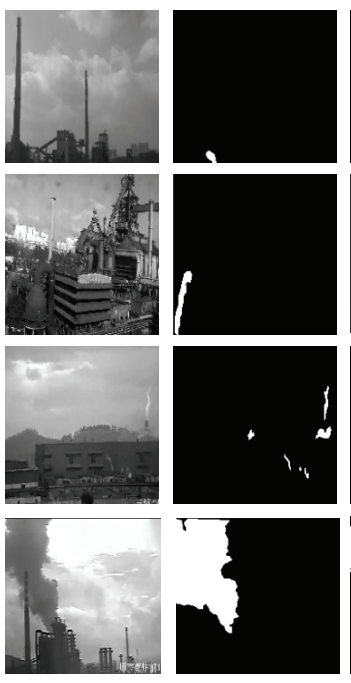

a. 原图 b. 人工标记

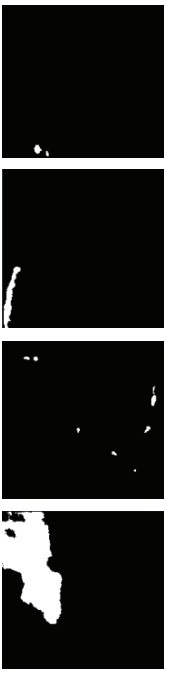

c. en-de ${ }^{[6]}$

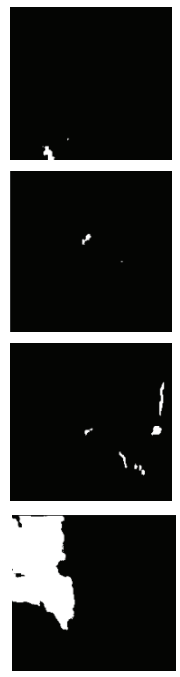

d. u-net ${ }^{[7]}$

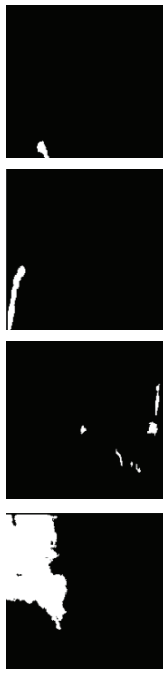

e. deeplab ${ }^{[28]}$

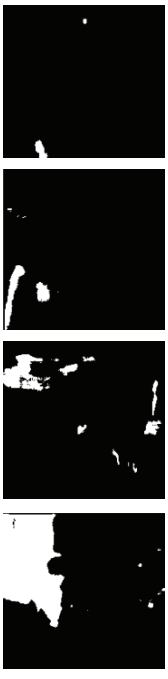

f. $\mathrm{FCN}^{[5]}$

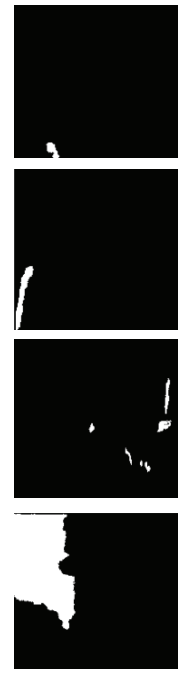

g. $\mathrm{mFCN}-\mathrm{s}$

图 13 干扰(云)场景分割结果对比

表 4 不同模型 IoU 指标对比

\begin{tabular}{lccccc}
\hline \multicolumn{1}{c}{ 模型 } & 易辨 & 小区域 & 薄烟 & 多目标 & 干扰 \\
\hline en-de $^{[6]}$ & 67.15 & 57.11 & 68.10 & 70.93 & 64.08 \\
u-net $^{[7]}$ & 78.95 & 69.53 & 72.98 & 74.93 & 68.62 \\
deeplab $^{[28]}$ & 81.39 & 74.86 & 71.61 & 76.13 & 76.47 \\
FCN $^{[5]}$ & 83.01 & 76.11 & 75.30 & 78.55 & 71.58 \\
mFCN-s & 84.16 & 78.61 & 76.79 & 78.92 & 78.89 \\
\hline
\end{tabular}

表 5 不同模型 $\boldsymbol{F}_{1}$ 度量指标对比

\begin{tabular}{lccccc}
\hline \multicolumn{1}{c}{ 模型 } & 易辩 & 小区域 & 薄烟 & 多目标 & 干抗 \\
\hline en-de $^{[6]}$ & 80.50 & 71.33 & 81.74 & 83.60 & 78.93 \\
u-net $^{[7]}$ & 88.32 & 73.78 & 84.83 & 86.19 & 80.39 \\
deeplab $^{[28]}$ & 89.77 & 86.04 & 83.81 & 86.50 & 85.93 \\
FCN $^{[5]}$ & 90.75 & 86.58 & 86.07 & 88.23 & 83.41 \\
mFCN-s & 91.44 & 88.26 & 87.08 & 88.38 & 88.15 \\
\hline
\end{tabular}

表 6 不同模型查准率指标对比

\begin{tabular}{lccccc}
\hline \multicolumn{1}{c}{ 模型 } & 易辨 & 小区域 & 薄烟 & 多目标 & 干抗 \\
\hline en-de $^{[6]}$ & 87.07 & 76.13 & 84.83 & 87.50 & 81.45 \\
u-net $^{[7]}$ & 91.92 & 85.23 & 88.66 & 90.03 & 81.24 \\
deeplab $^{[28]}$ & 92.34 & 89.78 & 89.11 & 90.61 & 89.76 \\
FCN $^{[5]}$ & 90.13 & 82.44 & 83.68 & 86.69 & 78.44 \\
mFCN-s & 93.10 & 89.46 & 89.50 & 90.90 & 89.33 \\
\hline
\end{tabular}

表 7 不同模型查全率指标对比

\begin{tabular}{lccccc}
\hline \multicolumn{1}{c}{ 模型 } & 易辨 & 小区域 & 薄烟 & 多目标 & 干扰 \\
\hline en-de $^{\left[{ }^{[}\right]}$ & 74.85 & 67.11 & 78.86 & 80.03 & 76.55 \\
u-net $^{[7]}$ & 84.99 & 80.83 & 81.32 & 82.66 & 79.56 \\
deeplab $^{[28]}$ & 87.34 & 82.59 & 79.11 & 82.74 & 82.42 \\
FCN $^{[5]}$ & 91.38 & 91.17 & 88.60 & 89.82 & 89.05 \\
mFCN-s & 89.84 & 87.08 & 84.79 & 85.98 & 87.01 \\
\hline
\end{tabular}

到云的干扰较严重(图 13f 第 1 第 4 行), $\mathrm{mFCN}-\mathrm{s}$ 在抗干扰和分割结果的完整性上表现更优.

综上, en-de 方法在烟尘分割完整性和小区域 烟尘识别方面表现不佳, 其采用的编码-解码结构, 在精细度方面应优于 FCN 等方法, 但因为烟尘是 边缘粗粘的非刚体, 且实验所用数据分辨率不高, 在精细度方面区分并不明显. u-net 方法易出现空 洞, 出现了对靠近图像边缘的烟尘区域识别不准 确的情况, 原因是相比其他模型, 其池化层最少, 且没有较大尺度的卷积操作, 以至于感受野不如 其他几种模型. FCN 在对于烟尘分割的完整性以及 抗干扰性方面表现不佳. deeplabv3+模型结合了更 多的改进思路, 包含残差结构与多尺度空洞卷积 结构, 相比 en-de, u-net 和 FCN 的烟尘分割结果更 准确, 但是在烟尘分割结果的完整性方面有待提 高. 本文 $\mathrm{mFCN}-\mathrm{s}$ 采用了多尺度卷积提高模型对烟 尘的特征提取能力, 又通过动态权重代价函数增 强对分类不准确目标的训练, 烟尘分割的结果更 准确，在对比模型中更接近于人工标记的结果.

在量化指标方面, $\mathrm{mFCN}-\mathrm{s}$ 的 5 个场景下的 IoU 均优于其他 4 种模型, 与改进前的 FCN 模型相比, 在干扰场景上最多有 $7.31 \%$ 的提升. 对于综合考虑 查全率和查准率的 $F_{1}$ 度量指标, 在 5 个场景下也 都高于其他 4 种模型, 比改进前最多提升了 3.6\%.

\section{4 结 语}

本文针对将 FCN 应用于工业烟尘图像分割时 准确度不高的问题, 采用了一种改进的全卷积网 
络模型，用多尺度卷积操作替换了原有的单一尺 度的卷积操作，用改进的代价函数替换了原有的 交叉熵代价函数. 本文提出模型的特点在于:

（1）使用多尺度的卷积核带来了不同大小的 感受野, 融合了不同尺度的特征, 增强了网络对工 业烟尘的特征提取能力.

（2）设计了带有动态权重的代价函数提高训 练的效率, 通过增强对分类不准确的点的训练, 进 一步提升网络对工业烟尘的分割性能.

为验证本文模型对工业烟尘图像分割的有效 性，将测试集按照易辨场景、薄烟场景、小区域烟 尘场景、多目标烟尘场景和干扰场景进行了分类, 在 5 个场景测试集上同 FCN 等 4 种图像分割模型 进行了对比测试, 通过 IoU, $F_{1}$ 度量、查准确率和 查全率 4 项指标进行量化测试. 实验结果表明，本 文模型比 FCN 在抗干扰性和分割完整性方面提升 明显，分割烟尘结果更为准确.

\section{参考文献(References):}

[1] Hsu Y C, Dille P, Sargent R, et al. Industrial smoke detection and visualization[R]. Pittsburgh: Carnegie Mellon University. School of Computer Science, 2016

[2] Xiao Zhaoxia, Chen Sheng. Review of image semantic segmentation[J]. Software Guide, 2018, 17(8): 6-8+12(in Chinese) (肖朝霞, 陈胜. 图像语义分割问题研究综述 $[\mathrm{J}]$. 软件导刊, 2018, 17(8): 6-8+12)

[3] Çelik T, Özkaramanlı H, Demirel H. Fire and smoke detection without sensors: image processing based approach[C] //Proceedings of the 15th European Signal Processing Conference. Los Alamitos: IEEE Computer Society Press, 2007, 2: 1794-1798

[4] Zhang Xiaomei, Wang Chengmei, Han Hu. Region based segmentation methods for soot image[J]. Computer Engineering and Applications, 2008, 44(13): 193-195+237(in Chinese) (张晓梅, 王诚梅, 韩琥. 基于区域的烟尘图像分割方法 [J]. 计算机工程与应用, 2008, 44(13): 193-195+237)

[5] Shelhamer E, Long J, Darrell T. Fully convolutional networks for semantic segmentation[J]. IEEE Transactions on Pattern Analysis and Machine Intelligence, 2017, 39(4): 640-651

[6] Badrinarayanan V, Kendall A, Cipolla R. SegNet: a deep convolutional encoder-decoder architecture for image segmentation[J]. IEEE Transactions on Pattern Analysis and Machine Intelligence, 2017, 39(12): 2481-2495

[7] Hu X G, Yang H G. DRU-net: a novel U-net for biomedical image segmentation[J]. IET Image Processing, 2020, 14(1): 192-200

[8] Lee C Y, Lin C T, Hong C T, et al. Smoke detection using spatial and temporal analyses[J]. International Journal of Innovative Computing, Information and Control, 2012, 8(7A): $4749-4770$
[9] Cheung S C S, Kamath C. Robust background subtraction with foreground validation for urban traffic video[J]. EURASIP Journal on Advances in Signal Processing, 2005, 2005(14): Article No.726261

[10] Wei Xubin, Wang Xiangjun. Smoke target detection based on accumulated differential image sequence and Gaussian background statistical model[J]. Journal of Astronautic Metrology and Measurement , 2007, 27(2): 58-61(in Chinese) (魏旭宾, 王向军. 基于累积差分图像和高斯背景统计模型 的烟尘目标检测 [J]. 宇航计测技术, 2007, 27(2): 58-61)

[11] Calderara S, Piccinini P, Cucchiara R. Smoke detection in video surveillance: a MoG model in the wavelet domain[C] //Proceedings of International Conference on Computer Vision Systems. Heidelberg: Springer, 2008: 119-128

[12] Gubbi J, Marusic S, Palaniswami M. Smoke detection in video using wavelets and support vector machines[J]. Fire Safety Journal, 2009, 44(8): 1110-1115

[13] Wang Wenzhe, Liu Hui, Wang Bin, et al. Image segmentation method of industrial fumes based on background modeling and feature matching $[\mathrm{J}]$. Transducer and Microsystem Technologies, 2018, 37(8): 37-39+42(in Chinese) (王文哲，刘辉，王彬，等. 基于背景建模与特征匹配的工业 烟尘图像分割方法 [J]. 传感器与微系统 2018, 37(8): 37$39+42)$

[14] Zhao Min, Zhang Wei, Wang Xin, et al. A smoke detection algorithm with multi-texture feature exploration under a spatio-temporal background model[J]. Journal of Xi'an Jiaotong University, 2018, 52(8): 67-73(in Chinese) (赵敏, 张为, 王金金, 等. 时空背景模型下结合多种纹理特征 的烟雾检测 [J]. 西安交通大学学报, 2018, 52(8): 67-73)

[15] Yuan F N, Zhang L, Xia X, et al. Deep smoke segmentation[J]. Neurocomputing, 2019, 357: 248-260

[16] Li Yingcheng, Qian Sainan, Zhu Xiange, et al. Application of convolutional neural networks in large-scale image classification[J]. Science of Surveying and Mapping, 2019, 44(6): 121-125(in Chinese)

(李英成, 钱赛男, 朱祥娥, 等. 卷积神经网络在大规模图像 分类中的应用 [J]. 测绘科学, 2019, 44(6): 121-125)

[17] Wang Wenxiu. Fu Yutian, Dong Feng, et al. Infrared ship target detection method based on deep convolution neural network[J]. Acta Optica Sinica, 2018, 38(7): 160-166(in Chinese) (王文秀, 傅雨田, 董峰, 等. 基于深度卷积神经网络的红外 船只目标检测方法 [J]. 光学学报, 2018, 38(7): 160-166)

[18] Deng Lin. Research on video smoke detection algorithm[D]. Chengdu: University of Electronic Science and Technology of China, 2015: 46-60(in Chinese) (邓林. 视频烟雾检测算法研究 [D]. 成都: 电子科技大学, 2015: 46-60)

[19] Wang Wenzhe. Research on target region segmentation method for industrial soot emission image monitoring[D]. Kunming: Kunming University of Science and Technology, 2018: 36-48(in Chinese)

(王文哲. 面向工业烟尘排放图像监测的目标区域分割方法 研究[D]. 昆明: 昆明理工大学, 2018: 36-48)

[20] Wang Sen, Wu Xing, Zhang Yinhui, et al. Image crack detection with fully convolutional network based on deep learning $[\mathrm{J}]$. Journal of Computer-Aided Design \& Computer Graph- 
ics, 2018, 30(5): 859-867(in Chinese)

(王森, 伍星, 张印辉, 等. 基于深度学习的全卷积网络图像 裂纹检测 [J]. 计算机辅助设计与图形学学报, 2018, 30(5): 859-867)

[21] Jiang Wentao, Zhang Chi, Zhang Shengchong, et al. Multiscale feature map fusion algorithm for target detection[J]. Journal of Image and Graphics, 2019, 24(11): 1918-1931(in Chinese) (姜文涛, 张驰，张晟羽，等. 多尺度特征图融合的目标检测 [J]. 中国图象图形学报, 2019, 24(11): 1918-1931)

[22] Szegedy C, Liu W, Jia Y Q, et al. Going deeper with convolutions[C] //Proceedings of the IEEE Conference on Computer Vision and Pattern Recognition. Los Alamitos: IEEE Computer Society Press, 2015: 1-9

[23] Szegedy C, Vanhoucke V, Ioffe S, et al. Rethinking the inception architecture for computer vision[C] //Proceedings of the IEEE Conference on Computer Vision and Pattern Recognition. Los Alamitos: IEEE Computer Society Press, 2016: 2818-2826

[24] Xu Xiao. Research on fast object segmentation and detection based on deep learning[D]. Beijing: University of Chinese
Academy of Sciences, 2016(in Chinese)

(徐霄. 基于深度学习的快速物体分割和检测方法研究 [D]. 北京: 中国科学院大学, 2016)

[25] Lin T Y, Goyal P, Girshick R, et al. Focal loss for dense object detection[C] //Proceedings of the IEEE Conference on Computer Vision. Los Alamitos: IEEE Computer Society Press, 2018: $2980-2988$

[26] Tan Shili, Lei Humin, Wang Pengfei. Design of tracking differentiator based on tangent sigmoid function[J]. Systems Engineering and Electronics, 2019, 41(7): 1590-1596(in Chinese) (谭诗利, 雷虎民, 王鹏飞. 基于正切 Sigmoid 函数的跟踪微 分器 [J]. 系统工程与电子技术, 2019, 41(7): 1590-1596)

[27] Zhou Zhihua. Machine learning[M]. Beijing: Tsinghua University Press, 2016: 30-32(in Chinese)

(周志华. 机器学习[M]. 北京: 清华大学出版社, 2016: 30-32)

[28] Chen L C, Zhu Y K, Papandreou G, et al. Encoder-decoder with atrous separable convolution for semantic image segmentation $[\mathrm{C}] / /$ Proceedings of the European Conference on Computer Vision. Heidelberg: Springer, 2018: 801-818 